\title{
Light Pollution Mapping from a Stratospheric High-Altitude Balloon Platform
}

\author{
Geza GYUK ${ }^{1 *}$, Jesus G. GARCIA ${ }^{1}$, Cynthia TARR ${ }^{1}$, Ken WALCZAK ${ }^{1}$ \\ ${ }^{1}$ Adler Planetarium, Chicago, Illinois, USA
}

Received 18 September 2020, Accepted 03 March 2021

\begin{abstract}
The NITELite (Night Imaging of Terrestrial Environments Lite) system is a method of collecting regional-scale light emissions data from a latex high-altitude balloon (LHAB) platform. An LHAB can reach altitudes of 25-30km from where the nighttime imaging is performed. LHAB missions are relatively low cost $(<\mathrm{US} \$ 2000 /$ flight $)$ and easy to repeat. A NITELite mission collects data with high resolution $(<10$ meters per pixel), color information (RGB) over a region of thousands of square kilometers. This system provides a new source of data for remote sensing of artificial light at night (ALAN) research, filling the data gap between low-altitude aircraft and satellite observations. Nighttime LHAB-based imaging can provide data to support fields of ALAN research such as observation of real-time variability, monitoring effects of seasonal changes and events of interest, and measuring angular dependence of ALAN sources. NITELite includes an imaging system, an inertial and positional recording on-board-computer, and an altitude control system. Preliminary results demonstrate the potential of this method for future ALAN research.
\end{abstract}

Keywords: NITELite, GONet, remote sensing, high altitude balloon, light pollution

\section{Introduction}

Light Pollution (LP) or Artificial Light At Night (ALAN) by its simplest definition is the contamination of the natural nighttime environment by light of anthropogenic origin. ALAN has been demonstrated to affect ecosystems and human health, as well as have a measurable impact on economic and environmental well-being[1-4]. Many studies over more than two decades have used remotely sensed data to shine a light on the sources, impacts and costs of light pollution[59]. Light pollution research has suffered in the past from a lack of data. After the public release in the 1990s of the first satellite data of the Earth at night from the Defense Meteorological Satellite Program (DMSP) Operational Linescan System (OLS) instrument, our understanding of the impacts of ALAN have grown, as reflected by the number of published papers since that time. Since the launch of the Visible Infrared Imaging Radiometer Suite (VIIRS) instrument on board the Suomi-NPP satellite in 2011, reliable quality data available for research has helped to advance our understanding of the impact of ALAN in a multitude of fields. Still, the need for better data with higher resolution, better color definition, and more on-demand availability can help progress our understanding of the effects of ALAN in a number of fields. A small number of aerial surveys, performed over targets of interest, have provided high resolution data. The utility of aerial surveys has limitations, however. In all these cases, evidence shows that in the field of ALAN research the improvement of available data leads to new understandings of its impacts. The NITELite system has the capacity to provide a new level of data for light pollution research.

\section{Remote Sensing for ALAN}

The two primary remote sensing methods used to acquire ground light emission data for ALAN research have been orbital missions and aerial surveys. Both methods offer advantages and disadvantages in the data they can provide. Satellite missions typically offer global or large-scale datasets and nightly or frequent reimaging. Aerial surveys excel at

*G. Gyuk, E-mail address: ggyuk@adlerplanetarium.org 
capturing high-resolution, color data on an urban scale. Recently, work has been done with unmanned aerial vehicles and large-scale research balloons[10,11].

\subsection{Orbital Missions}

Orbital data is dominated by two satellite missions: The DMSP and Suomi National Polar-orbiting Partnership (SuomiNPP). Data from the Operational Linescan System (OLS) instrument on the DMSP satellite have been collected since the 1970s and have been available to the public since 1992[12,13]. Although the data from the DMSP helped in the creation of the first global map of light emission it suffers from some limitations for research[14]. It provides only $5 \mathrm{~km} / \mathrm{px}$ resolution, a less than favorable 19:30 local time overpass time, a single panchromatic bandpass, and lacks stringent calibration[15]. The Visible Infrared Imaging Radiometer Suite (VIIRS) instrument on the Suomi-NPP satellite has been providing on-orbit data since soon after its launch in late 2011. VIIRS improved dramatically on the quality of data previously provided by the DMSP missions[16,17]. For the next-generation requirements of LP research, however, VIIRS data are still lacking, including: $742 \mathrm{~m} / \mathrm{px}$ resolution, a late 01:30AM local overpass time, single panchromatic bandpass imagery, and a "blue blindness" below 500nm that fails to detect significant emission from many types of outdoor LED lighting being used in modern municipal retrofitting projects[18].

Recent smaller scale satellite missions have demonstrated that MicroSats (10-100kg) or even CubeSats (1-10kg) can provide valuable data, as they can dedicate a mission and hardware to a single scientific purpose[19-22]. To date, the data from these missions are either only commercially available, limited in coverage, or collected primarily as a proof of concept. If this recent work is any indication, dedicated small satellite missions are soon to be the future of remote sensing for LP research.

DSLR images taken of the Earth at night by astronauts on board the International Space Station (ISS) are another source of orbital data. With high resolution $(\sim 5 \mathrm{~m} / \mathrm{px})$, three colors (broadband RGB) and good sensitivity, these ISS images have a wealth of use for research[23-25]. Unfortunately, they suffer from poor coverage (both temporally and geographically) or limited calibrated (both geometrically and photometrically).

\subsection{Aerial Surveys}

A small number of nighttime aerial surveys have been performed over urban centers. A few examples include flights over Berlin (Germany)[26], Birmingham (UK) [27] and Deventer (NL)*. These surveys have provided very highresolution color imagery unavailable by other methods. The data they produced have been used to investigate a number of aspects of nighttime lighting in urban areas, including source identification via spectral information, the relationship of land use to lighting, and quantification of urban light emission by lighting class. Often aerial surveys have the advantage of being performed in direct coordination with or via the funding of the researchers involved and are hence directly targeted in line with scientific goals of ALAN research. Unfortunately, aerial surveys can be very costly and time prohibitive. A single aerial survey of a mid-sized or large urban area can cost in the tens to hundreds of thousands of USD. Difficulties in the logistics of planning dedicated aerial flights can constrain the capacity of these surveys for longitudinal studies.

\subsection{High Altitude Balloon Missions}

High-altitude balloons (HABs) have been used for decades for atmospheric research, astrophysical research, and remote sensing[28-30]. Large research balloons are capable of carrying over 1 ton of payload and can reach altitudes well over $30 \mathrm{~km}$. The mass of these HAB payloads also makes them quite stable. Space for experiments is often available on $\mathrm{HAB}$ missions launched by national space agencies[31]. A recent experiment used a research-scale HAB mission to image light emission in Canada[32]. While these large missions can play a valuable role in remote sensing of LP, quite often their overflight paths are constrained by the predetermined launch location, or by the needs of a primary experiment. In addition, a single large research HAB flight can cost 1 million USD, a cost beyond the reach of a typical LP research program.

Over the past two to three decades the number of small (500-2000g) latex high altitude balloon (LHAB) missions has grown, driven by private individuals, educators and students due to the ease of setup, shallow learning curve and relatively low cost of performing a mission. Latex balloon missions can be flown with low initial investments and per-flight costs in the $\$ 100$ 's to low $\$ 1,000$ 's range.

\footnotetext{
* Accessed at https://www.arcgis.com/apps/Viewer/index.html?appid=81204cec708945dba62b257121375c05
} 
For over a decade the Far Horizons program at the Adler Planetarium has used LHABs for education and research. NITELite was developed as a precursor and technological readiness experiment for the NITESat orbital mission concept [33]. LHABs for light pollution imaging offer some unique advantages over other sources of remotely sensed LP data. Compared to most orbital data, NITELite data has much higher resolution, provides color information, can be flown on demand, and can image over the course of a night. Compared to aerial surveys, NITELite is an order of magnitude cheaper, more easily repeatable, and can provide a much larger mapped footprint.

The challenges of LHAB missions include reaching desired altitude, providing a controlled descent, reducing rotational motion, choosing launch location based on wind pattern predictions, and choosing an adequate power source for operating in sub-zero temperatures. Weather and payload retrieval can also pose problems at times. The rest of this paper describes the NITELite system and some preliminary data, illustrating what is possible with this type of LHAB system.

\section{NITELite System Architecture}

The LHAB NITELite Mission is composed of a mix of custom systems as well as commercial off-the-shelf (COTS) products that are easily accessible for duplication of the payload. In order to successfully achieve a nighttime survey of light pollution, several factors were considered, such as rotational stability during imaging, imaging cameras with high sensitivity at low-light and an acceptable spectral response, an on-board computer capable of capturing spatial information as well as atmospheric conditions during flight, and an adequate power source to withstand sub-zero temperatures.

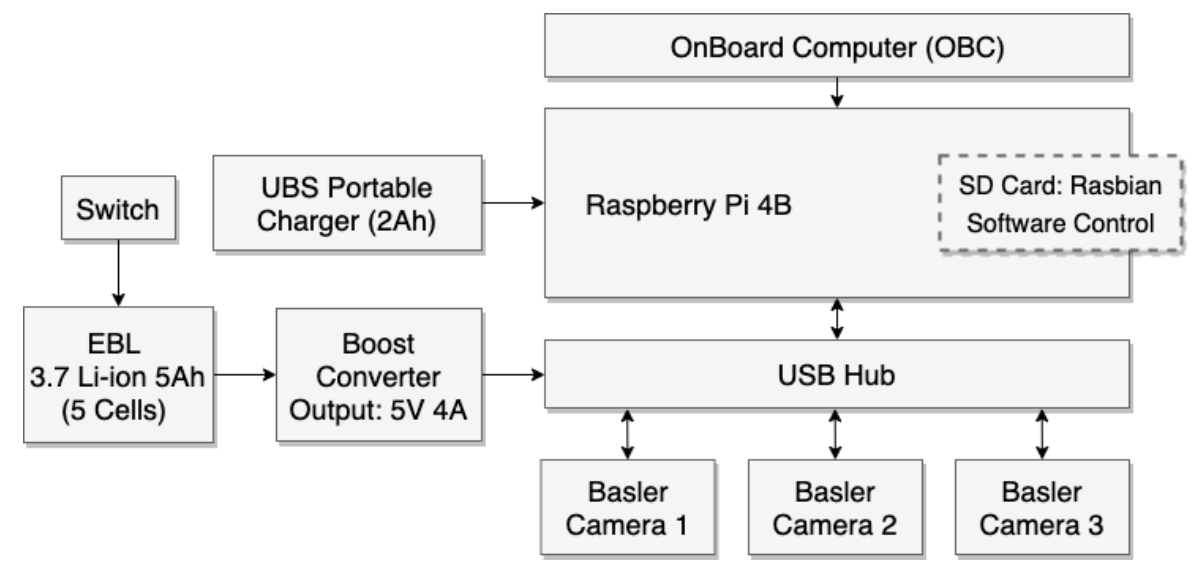

Figure 1: Block diagram of electronic equipment inside NITELite payload, which runs autonomously and is responsible for capturing $\sim$,000 images per hour.

In response to these constraints, the payload is composed of an Altitude Control System (ACS) capable of controlling ascent velocity/reaching neutral buoyancy, a Raspberry Pi-controlled Imaging System capable of full color imaging in low-lighting, an On-Board Computer (OBC) to capture spatial information and atmospheric conditions, and a thermallyinsulated battery compartment supplying sufficient current to power all equipment under cold environments. Figure 1 details the systems and their interactions. We discuss each of these systems in more detail in the following sections.

\subsection{Imaging System}

The camera system of the NITELite mission uses three industrial CMOS sensors, each fitted with a $25 \mathrm{~mm} \mathrm{f} / 1.4$ megapixel rated camera lens. The Basler acA1920-40uc cameras utilize an IMX249 sensor, producing a 1920x1200 Bayer tiled RGB image. This lens-sensor combination produces a $23.4^{\circ} \times 14.8^{\circ}$ field of view. The pixel size is $5.4 \mu \mathrm{m}$, corresponding to an average angular scale of $0.0123^{\circ} / \mathrm{pixel}$, giving a ground scale of about $5 \mathrm{~m} / \mathrm{px}$ at $25 \mathrm{~km}$ altitude. The three cameras are aligned, with one nadir pointing camera flanked by two off-axis cameras tilted to create a continuous strip of images with sufficient overlap, providing a $7 \mathrm{~km} \times 30 \mathrm{~km}$ image swath at altitude (see:

).

The imaging sequence consists of rapidly capturing 5 uncompressed RAW images with 50-ms exposures from each camera followed by a 100-ms compressed TIFF that is used as a thumbnail reference. The 50 milliseconds for exposure was chosen to balance the need for long exposure times to capture low light environments with blurring caused by the small inherent motion of the imaging platform. The three cameras are set to image at near synchronous timing every $\sim 7$ seconds. A one-hour imaging routine produces 465 complete swaths. 
With a 1/50-s exposure the IMX249 is theoretically capable of detecting surfaces illuminated (at $\sim 5$ sigma) as low as 0.5 lux, corresponding to point sources as dim as $\sim 10$ lumens. In practice, we do not typically achieve this level of performance but can estimate a sensitivity of $10-9 \mathrm{~W} / \mathrm{cm}-2 / \mathrm{sr}$ from test flights based on observation of reflectance of lunar illumination from the ground.

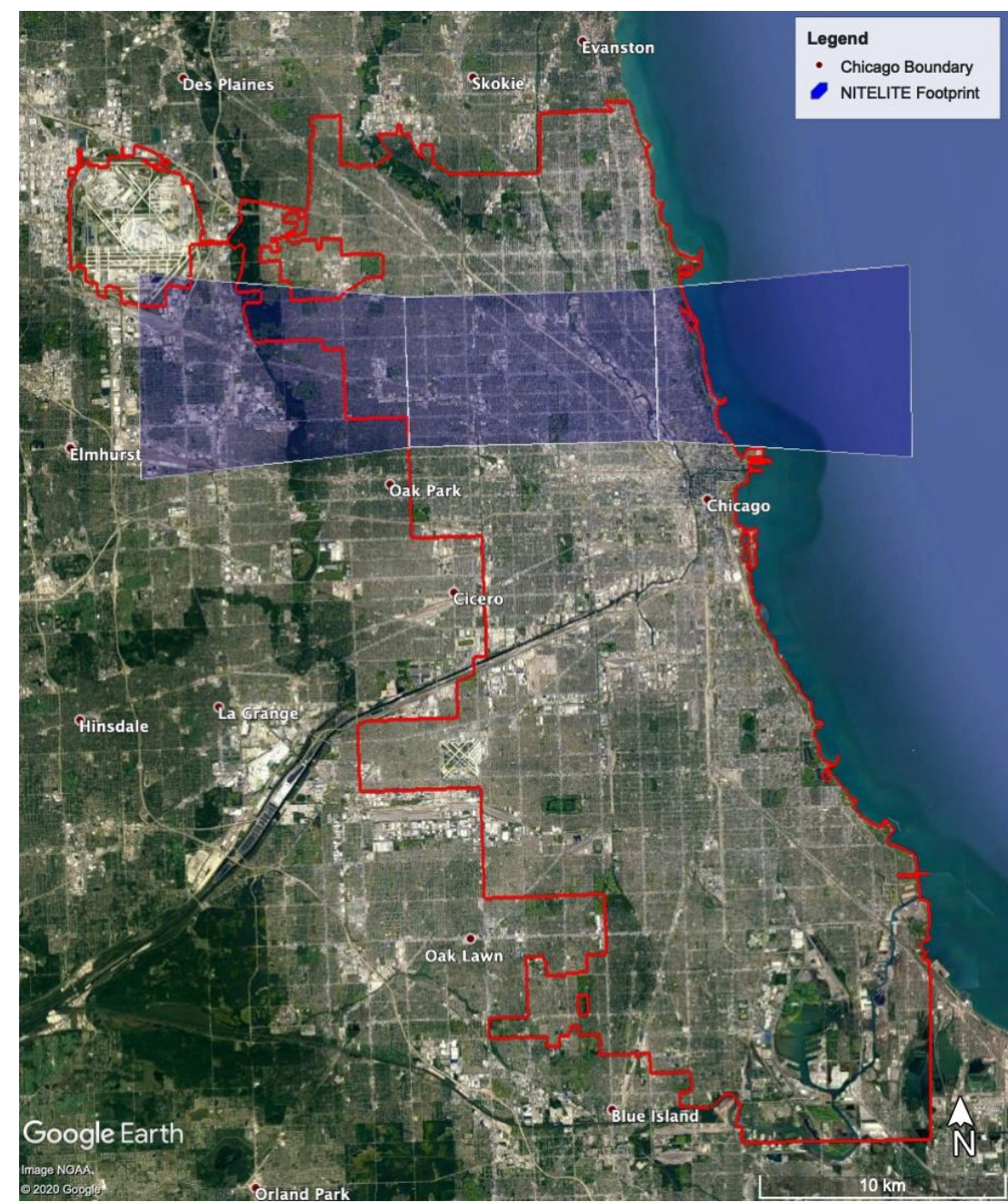

Figure 2: Schematic diagram of the $7 \mathrm{~km}$ x $30 \mathrm{~km}$ imaging footprint (blue) seen by the NITELite system at an altitude of $25 \mathrm{~km}$, superimposed on Chicago (city borders outlined in red) for context. (Map Data: Google/NOAA)

\subsection{Altitude Control System (ACS)}

Based on prior LHAB non-controlled ascent payload missions, it was observed that motion blur due to rotation was a severe constraint on the possible exposure times and hence imaging sensitivity at night. The ability to minimize rotation of the payload is crucial for capturing images of $50 \mathrm{~ms}$ or longer.

LHAB missions ascend from the buoyancy of a lifting gas - typically helium. As the balloon ascends and the pressure drops, the lifting gas expands and stretches the latex. The motion of the balloon envelope through the still air (typically at speeds $\sim 5 \mathrm{~m} / \mathrm{s}$ ) distorts the balloon in chaotic ways, pumping energy into the balloon-payload system. The payload z-axis rotational modes are particularly susceptible to excitation, and rotation speeds of tens of degrees per second are common. In addition, this rotation is very chaotic, changing on timescales of seconds, making correcting systems extremely difficult. The best option is to reduce the source of the rotation, vertical motion through the air, in the first place.

The Altitude Control System (ACS) was designed to solve this problem. The ACS is a cylindrical programmable venting system that is inserted into the neck of the balloon after it is filled for flight. At a preprogrammed altitude determined by wind models and desired imaging target - the ACS opens, allowing the helium to flow out. As the helium is released the ascent velocity slows. An on-board pressure sensor measures the change in altitude. Once the ACS detects 
the ascent has stopped, it closes the valve to maintain neutral buoyancy. During the period of neutral buoyancy, the payload and balloon are stable enough for nighttime imaging. An additional benefit is that by venting, and hence preventing the balloon from bursting, we can extend the length of time at altitude and for the imaging survey.

We can calculate the maximum allowable rotation rate needed to produce quality imagery. Including overlap, the center to center pixel distance between the nadir camera and the side pointing cameras is approximately 1500 pixels. Since the exposure time is $50 \mathrm{~ms}$, we calculate that the maximum rotation rate in the Z-axis to move a point source one pixel is $1 \%$. Thus, $\leq 0.5 \%$ will result in $<50 \%$ cross pixel blurring at the edge of the image for the nadir camera. Using the Inertial Measurement Unit (IMU) data provided by the OBC (Section 3.4) for each image, we can filter usable $\left(<1^{\circ} / \mathrm{s}\right)$, useful
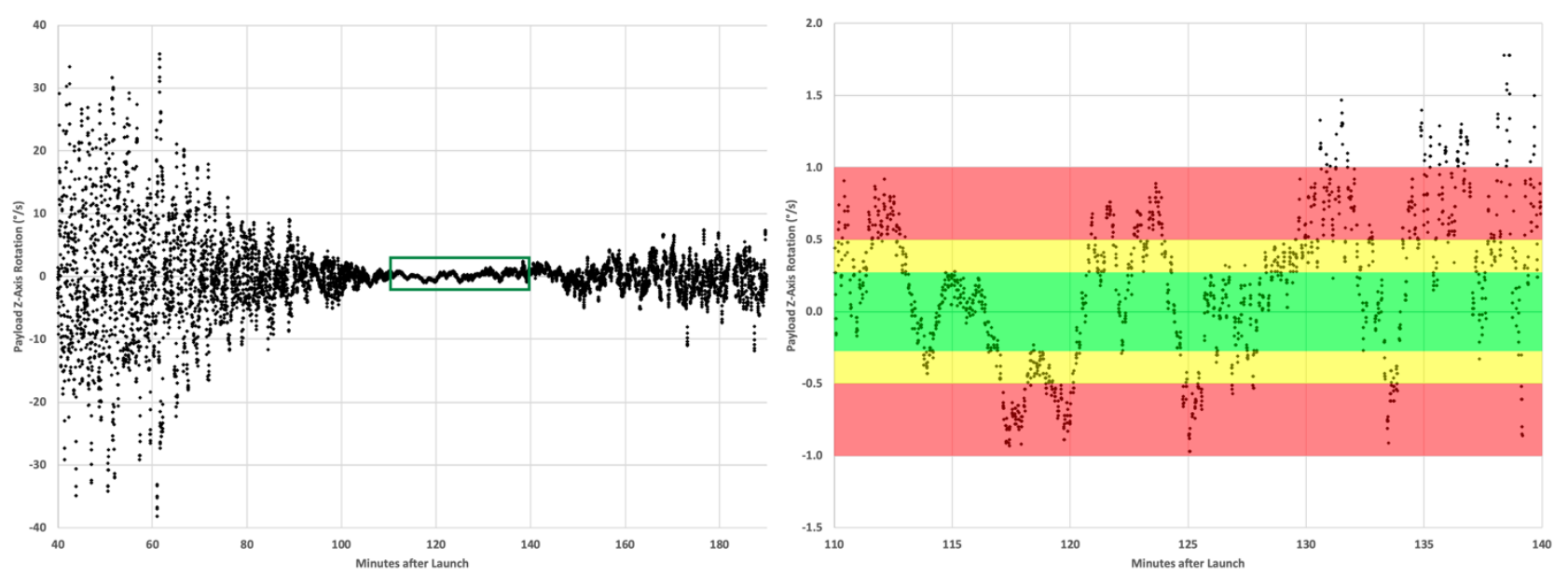

Figure 3: Left: Payload rotation velocity as a function of time with use of the Altitude Control System. The ACS began venting 80 minutes after launch and achieved neutral buoyancy at $\sim 100$ minutes. Right: Close-up of the neutrally buoyant period (green box in the left panel) with rotational speeds within $0.25,0.5$, and $1 \%$ s highlighted (green, yellow, and red bands, respectively).

$(<0.5 \%$ s) and high quality $(\leq 0.25 \% \mathrm{~s})$ images. Examining Figure 3 we see that, within the 60 -minute window of neutral buoyancy, $90 \%$ of images fall within $1 \%$ s, $67 \%$ within $0.5 \%$, and $36 \%$ within $0.25 \%$ s. An auxiliary rotation correction system to further reduce these rotation rates was considered but rejected as too technically demanding: vibrations from such a system were likely to cause greater rotational excitations than they corrected.

The ACS is composed of a hard shell constructed of PVC, within which is an electronics bay consisting of an Actuator, Teensy Microcontroller, an altimeter, and several power-regulating devices. The ACS is powered by four 3.7V lithium batteries that supply a continuous current of up to $180 \mathrm{~mA}$. Thermal control is provided by a heated layer of nichrome wire embedded in Kapton tape and powered by two 9V batteries. The ACS is wrapped by a final layer of low-density foam insulation and fiberglass insulation, which is exposed to the environment. Inside the PVC shell, all electronic components are required to function down to at least $-10^{\circ} \mathrm{C}$. The ACS has been lab-tested for correct function in both lowtemperature and vacuum environments. In addition, an extensive series of flights has proven the ACS in the field.

\subsection{On-Board Computer $(\mathrm{OBC})$}

To be able to identify, sort, geolocate, and postprocess the images, metadata is required. The On-Board Computer $(\mathrm{OBC})$ provides a feed of information to the imaging computer to tag images as they are recorded. That information includes latitude and longitude of the ground track, altitude, and a timestamp from a GPS unit. An Inertial Measurement Unit (IMU) provides instantaneous state data. This includes 3-axis acceleration, rotational data, and pointing data from an accelerometer, gyroscope, and magnetometer, respectively. This data (as demonstrated in Section 3.3 above) is used as a filter for image quality as well as assisting in the process of geolocating the imagery. The IMU collects data at a rate of $7 \mathrm{~Hz}$. A pressure sensor and temperature sensors are also part of the OBC system.

\subsection{Payload Infrastructure}

An LHAB research payload is exposed to wildly varying conditions: from humid and hot cornfields to the bone dry, low temperature/pressure, and high radiation levels of near-space. The NITELite payload is designed to overcome these conditions and perform the tasks required for light pollution surveys. The NITELite payload box is composed of a lowdensity foam insulator, which inhibits rapid changes in temperature and reduces the total mass of the payload. Apart from its protective insulation, the payload is encased in a layer of water-resistant ripstop material to allow any large amounts 
of humidity (cloud droplets) to easily roll-off the payload. Additionally, the payload has a layer of light-weight aluminum to reduce radio-frequency (RF) emissions from the onboard equipment. This layer greatly reduces interference with onboard transceivers.

The imaging control system is composed of a Raspberry Pi 4B, which consumes $\sim 650 \mathrm{mAh}$ during operations. This microprocessor is powered by a USB Portable Charger that can deliver $2 \mathrm{~A}$ of continuous current and has a total capacity of $23 \mathrm{Ah}$. The Basler cameras are connected to a USB hub, which is connected to a USB 3 port on the Raspberry Pi. This USB hub is powered by a set of Lithium-Ion batteries with total capacity of $25 \mathrm{Ah}$. The cameras and USB hub consume $1.56 \mathrm{~A}$ of continuous current during imaging. Finally, the On-Board Computer is connected directly to the USB port on the Raspberry Pi. The OBC only consumes about $180 \mathrm{~mA}$ of continuous current.

The payload infrastructure also includes two low-power, ham radio-based tracking systems that allow the flight crew to follow the flight, track the eventual descent, and retrieve the payload after landing.

\section{Mission Operations}

The details and logistics of an LHAB flight are important to understanding the advantages and limitations of LHAB mapping of LP. The average mission follows the following typical flight profile. After launch, preferably near the time of sunset, the mission ascends at a vertical velocity in the range of 4-6 m/s. To a small degree the ascent speed can be adjusted by regulating the quantity of helium used, but it is remarkably robust against variation in payload mass. After about 90 minutes of ascent, when the preprogrammed target altitude $(24-27 \mathrm{~km})$ is being neared, the ACS opens to vent helium. After 15-20 minutes, neutral buoyancy is achieved and the ACS closes the vent after detecting no significant change in pressure over a short sample time. At the imaging altitudes the wind velocities are quite low and stable. This begins the imaging phase of the mission. The length of time for imaging can be programmed into the ACS. The very low temperatures at this altitude are far below the operational specifications for the mechanical and power systems of the ACS. Although insulation and a heating system is part of the ACS, data from flight tests have suggested limiting the maximum dwell time to 90 minutes. Once the imaging phase is completed, the ACS will open the vent again to begin descent. Helium will vent out until the overpressure caused by the elastic relaxation of the balloon reaches an equilibrium. In flight tests the descent velocity stabilized between $2.5-3.2 \mathrm{~m} / \mathrm{s}$. With these parameters, a mission with a one-hour imaging phase would result in a total flight time of 250-300 minutes. Landing is typically in an agricultural setting and permission of the landowners is required for retrieval of the NITELite system the next morning.

\subsection{Flight Path and Predictions}

A brief understanding of the structure of our atmosphere is helpful in understanding the requirements of a NITELite flight. The troposphere is the layer of our atmosphere from the surface to $6-20 \mathrm{~km}$. It is in this layer that virtually all weather occurs. Regardless of season, in the middle latitudes, the temperature quickly drops and at the top of the troposphere it can reach -40 to $-50^{\circ} \mathrm{C}$. Some of the fastest winds in the lower atmosphere occur within this layer with velocities in excess of $200 \mathrm{~km} / \mathrm{h}$ common in middle latitudes. Winds can also be highly variable with altitude in the troposphere (see Figure 4), causing vertical wind shear, which transfers energy to the motion of the balloon/payload system. 


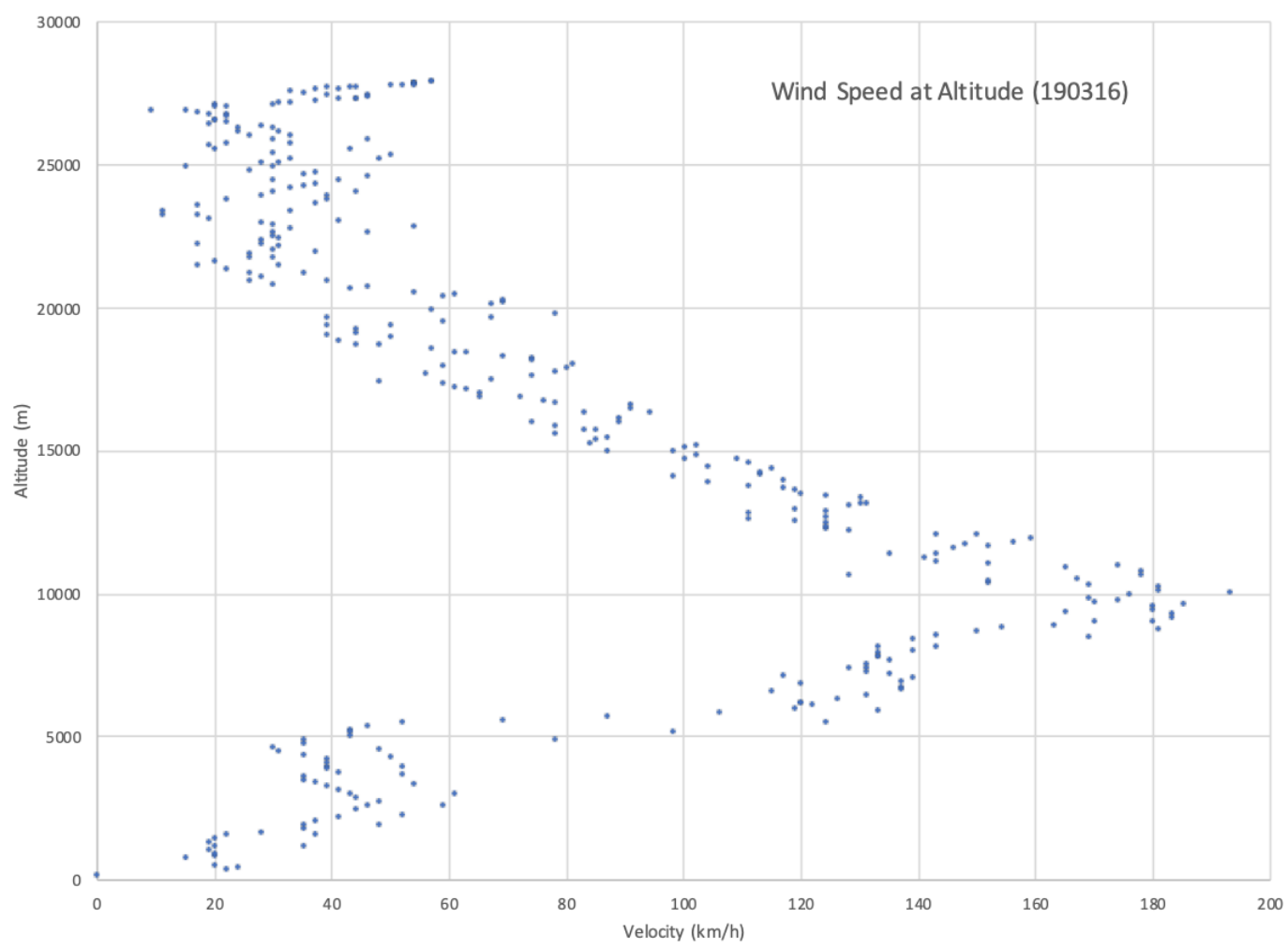

Figure 4: Wind speed recorded during flight as a function of altitude for a typical LHAB flight. Note the extremely high wind speeds at $10 \mathrm{~km}$ and the high wind shear below $20 \mathrm{~km}$.

The tropopause is a transition layer between the troposphere and stratosphere. In the tropopause there are often very turbulent flows whose intensity depends on the season and regional wind patterns. Above the tropopause is the stratosphere extending up to $50 \mathrm{~km}$. The temperature in the stratosphere rises as altitude increases. This inverted temperature profile prevents convection, making the stratosphere a relatively stable zone with low velocity winds. It is for these reasons that flying above the tropopause is strongly preferred to image effectively at night.

Since an LHAB has no active control, the wind directions and velocities throughout the ascent to the maximum altitude are what determine its imaging location. Targeting a region of interest for NITELite imaging requires predicting the path the balloon and payload will take and, from that prediction, determining a launch location. Tools that use Global Forecast System (GFS) wind models can predict the expected balloon path. The Cambridge University Spaceflight Landing Predictor (https://predict.habhub.org/) is a popular and very user-friendly method of flight prediction. It is a useful tool for a first-order determination of upcoming flight opportunities. It can generate predicted paths beginning 180 hours from launch time. Unfortunately, the software does not allow for a variable ascent profile that the NITELite ACS system requires. Balloon Prediction software created by the Ozone and Water Vapor Group within NOAA's Global Monitoring Division (https://www.esrl.noaa.gov/gmd/ozwv/wvap/sw.html) can be customized with variable ascent and descent profiles. Both these tools output KML files which are helpful for launch, tracking and retrieval planning.

The track of flights varies by region and season. In the Midwestern US, tropospheric winds are predominantly from the west to the east throughout the entire year. Winter winds are on average faster than those in the summer. The stratospheric winds are heavily dependent on the season. In middle latitudes, stratospheric winds in the summer (June to early September) are counterflowing (in the case of the Midwestern US - east to west). A transition occurs at the beginning and end of that period when the stratospheric winds begin turning to eventually settle into a west to east direction through the winter. This transition period can occur over a period of less than a month.

\subsection{Restrictions}

Ground conditions play an important role in a launch. In particular, the launch location is dependent upon the predicted path, which can vary greatly from day to day. In addition, local weather (e.g., thunderstorms) can scrub a launch for legal or technical reasons, even if the weather over the target is clear. 
Many countries have regulations guiding the use of HABs. They are typically regulated and controlled through each nation's aviation authority. In the United States, LHAB flights are regulated by the Federal Aviation Administration. These regulations primarily focus on mass limits, notification requirements, safety requirements, and restrictions.

\section{Data Pipeline}

Data from the imaging system is provided in the form of uncalibrated, 12-bit, raw Bayer-tiled 1900×1200-pixel images with an accompanying timestamp. Figure 5 shows a typical example over rural Indiana, including the towns of Culver, Monterey, and Hartz Lake. The region shown is approximately $10 \mathrm{~km}$ across. In comparison is the same region from resampled VIIRS DNB data. The wealth of added detail and color information is immediately obvious. A typical flight may contain hundreds of such images.
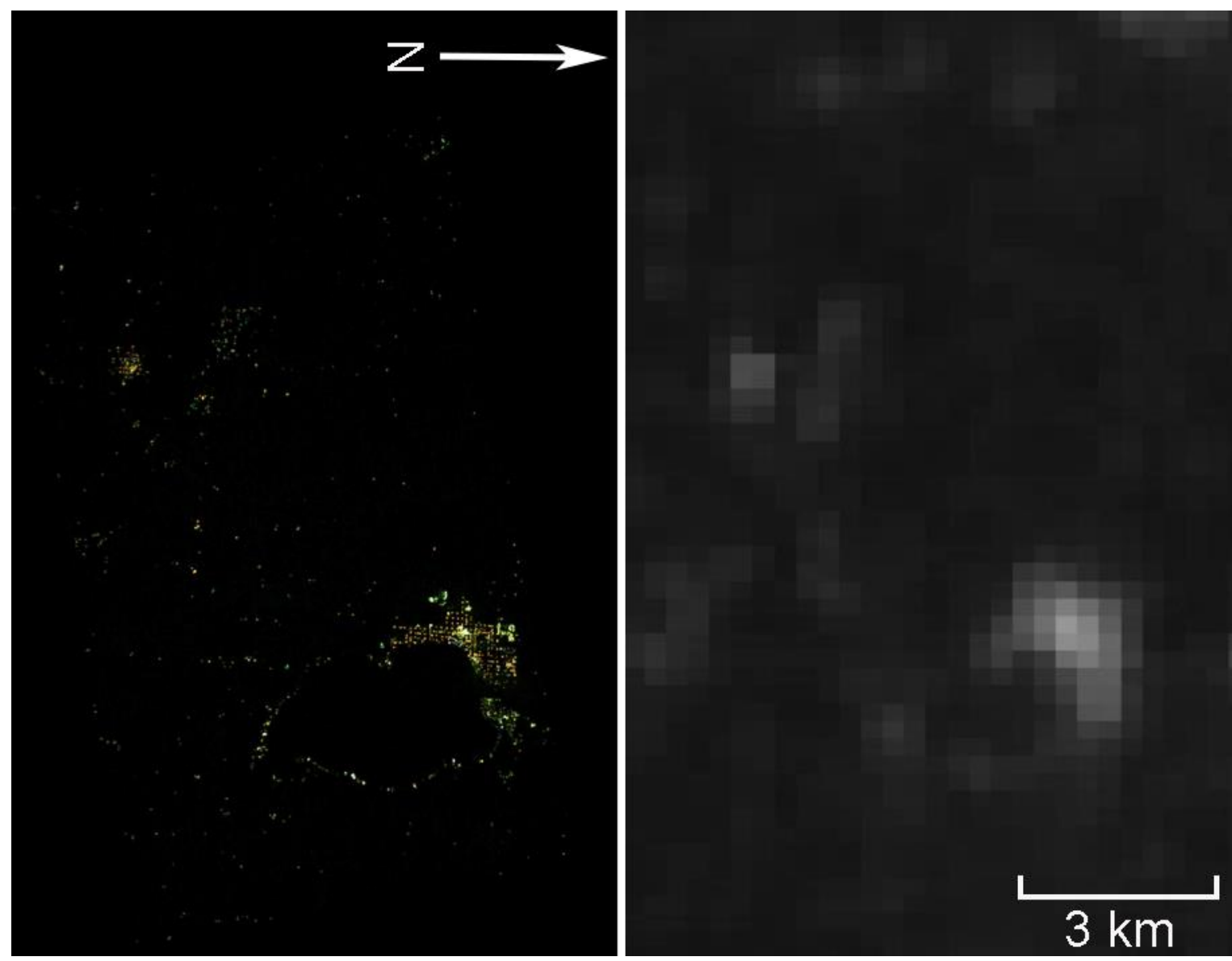

Figure 5: Region of rural Indiana, including the towns of Culver (bottom right) and Monterey (mid left). Left image is from NITELite, right image is from the VIIRS DNB (Image: NOAA).

We have developed a semi-automated, Python-based "pipeline" for analyzing the data. The first step of our analysis pipeline is to reduce the spatial resolution by a factor of two, grouping four pixels (RGGB) into one super pixel with full color information. While this gives up resolution, it has the advantage of being simple and easily computed.

The next step is georegistration. Metadata from the OBC subsystem is used to approximately locate and orient the images on the ground. At the current time, these approximate locations and orientations must then be adjusted by hand. This is done by visually identifying a set of unique light sources in both the nighttime images and on Google Earth daylight images of the same area. By extracting longitude and latitude from the daytime images and assigning them to the corresponding features of the nighttime images, a full 8 parameter perspective transformation,

$$
(x, y) \rightarrow\left(\frac{a x+b y+c}{g x+h y+1}, \frac{d x+e y+f}{g x+h y+1}\right)
$$


can be carried out transforming latitude and longitude to pixel coordinates. Typically, we find at least a dozen corresponding features spread over the image so the perspective transformation is robustly overdetermined. By recalculating with randomized points left out, a measure of the positional error introduced can be calculated. This is typically less than a pixel. Inverse transformations taking pixels to geographic coordinates are straightforward to obtain.

Once all images of a particular location (a "stack") are georegistered to a common system, we select a "template" image from which light sources of interest can be identified. The coordinates of these sources are then transformed to geographic coordinates using equation (1). These geographic coordinates are then, for each image in the "stack", transformed back to image coordinates, giving an image coordinate for each source in each image. Finally, the light from each source is measured by summing the pixel values above background around each projected source position. Surface brightnesses can be similarly measured by selecting a bounding polygon and summing pixel values within the projected polygon. Luminosity measurements are performed independently for each band (R, G, and B). At this point in time we have elected not to transform images and stack them for full dynamic range, but rather to consider each image separately and transform the coordinates. (For full analysis in the future, the set of 5 images will be stacked with pixel summation after registration to expand the sensitivity of the system.) This reduces the complexity of our analysis at the cost of reducing the sensitivity we can achieve.

Figure 6 shows the results of this process.
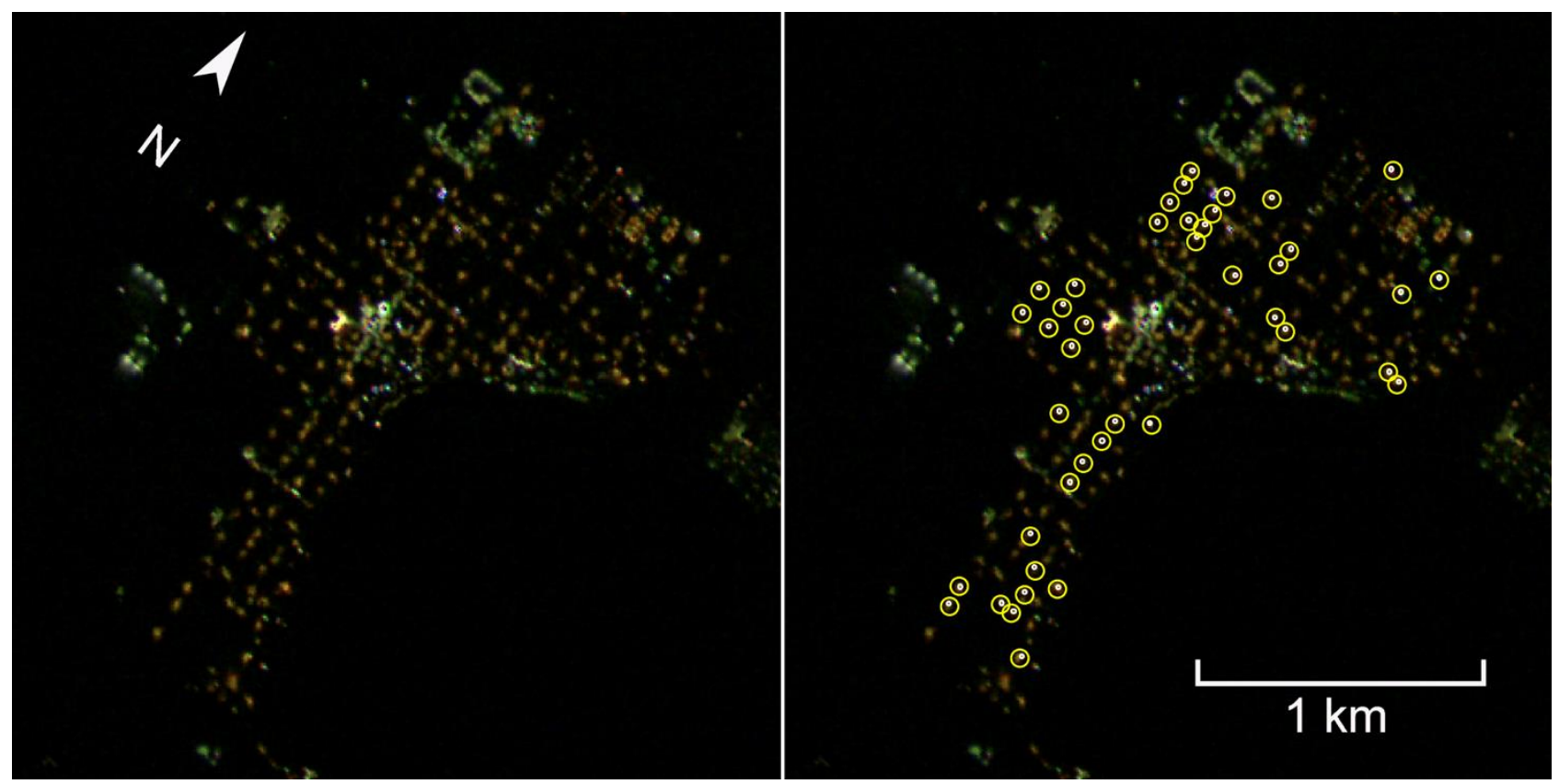

Figure 6: Detail of Culver, Indiana (left), and the same image (right) showing locations of sources propagated from the template image, which was taken approximately 15 minutes earlier and from a different angle.

\section{Preliminary Results}

\subsection{Photometric Stability}

To investigate the stability of our source luminosity pipeline we selected a set of 12 streetlight sources from our images of Culver. These images and sources were put through the standard pipeline. The results are shown in Figure 7.

It is immediately obvious that the sources undergo coordinated variability. A quick check back to the original images shows that dim epochs are directly correlated with clouds and vice versa. After adjusting for extinction presumed to be caused by atmospheric variation - since nearly all sources vary in concert with each other - we find that epoch-to-epoch variability for individual sources is well under the level of $\sigma \sim 10 \%$. We believe that much of this variability is due to issues with the photometric extraction and the small source size compared to the pixel scale. 


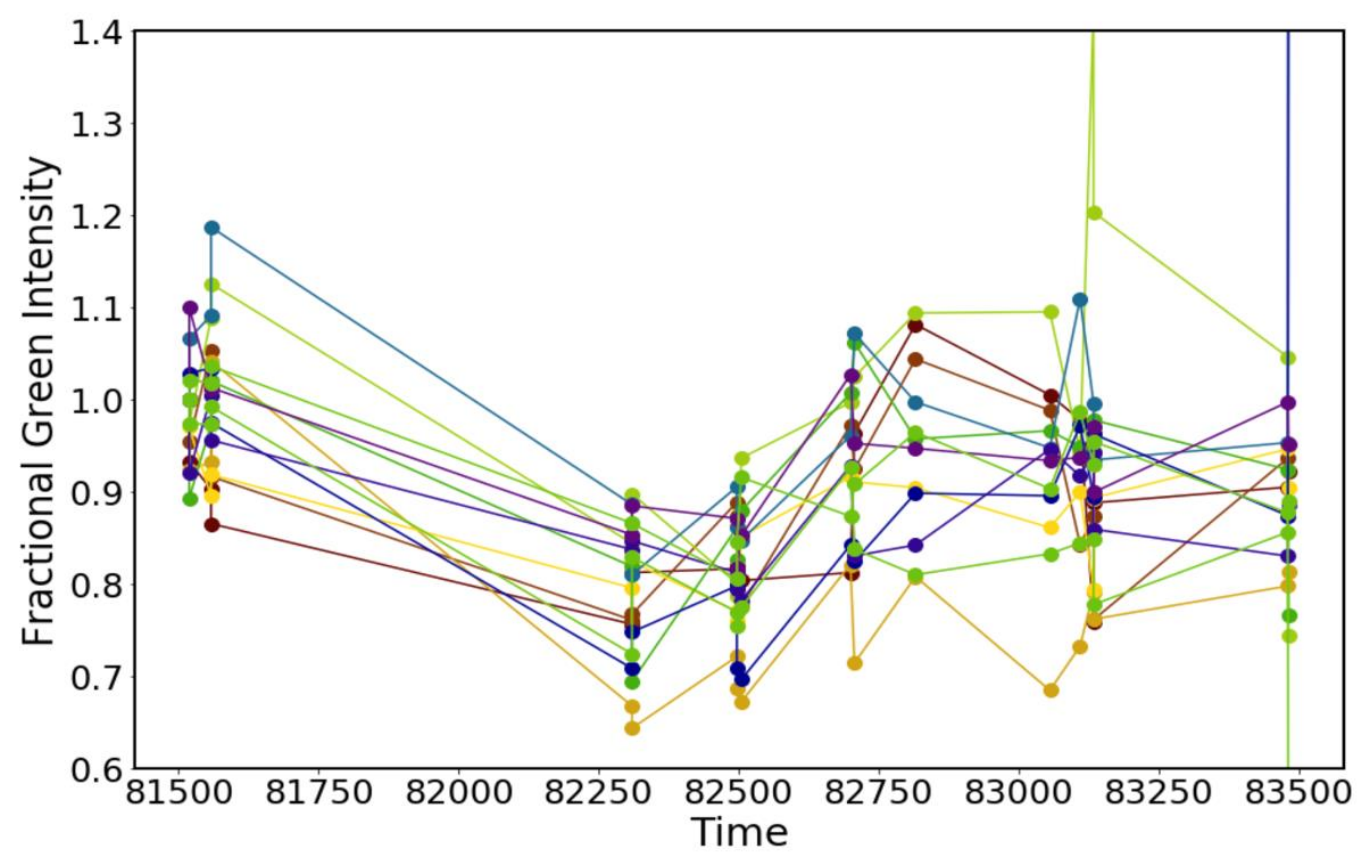

Figure 7: Uncalibrated light curves for 12 sources. When these are removed epoch-to-epoch variability is on the $10 \%$ level. The coordinated dips and rises have been visually identified being due to variable thin clouds. Outliers may be due to obscuration (low outliers) or high incidence of ground reflection (high outliers).

\subsection{Source Classification}

Analysis from NITELite missions demonstrates its capacity to discriminate sources of artificial light by lamp type. Figure 8 shows the preliminary analysis of a subset of light sources near Culver in the town of Monterey, Indiana, and the neighboring Hartz Lake "subdivision" (Figure 6). A series of 115 nadir images were obtained from an altitude of approximately 25,000 m over a period of 15 minutes (see Fig. 1 for example image). During this period the change in the angular direction to the sources was minimal, reducing the effects of changing viewing angle. These 115 images were grouped in "stacks" of 5, closely spaced in time. Light sources were centroided and photometry was performed on each image/source independently and then averaged within stacks. Shown are the color ratios of 60 sources in a single stack. There is clear separation between the lights in Monterey and the newer Hartz Lake residential subdivision. Further, visual

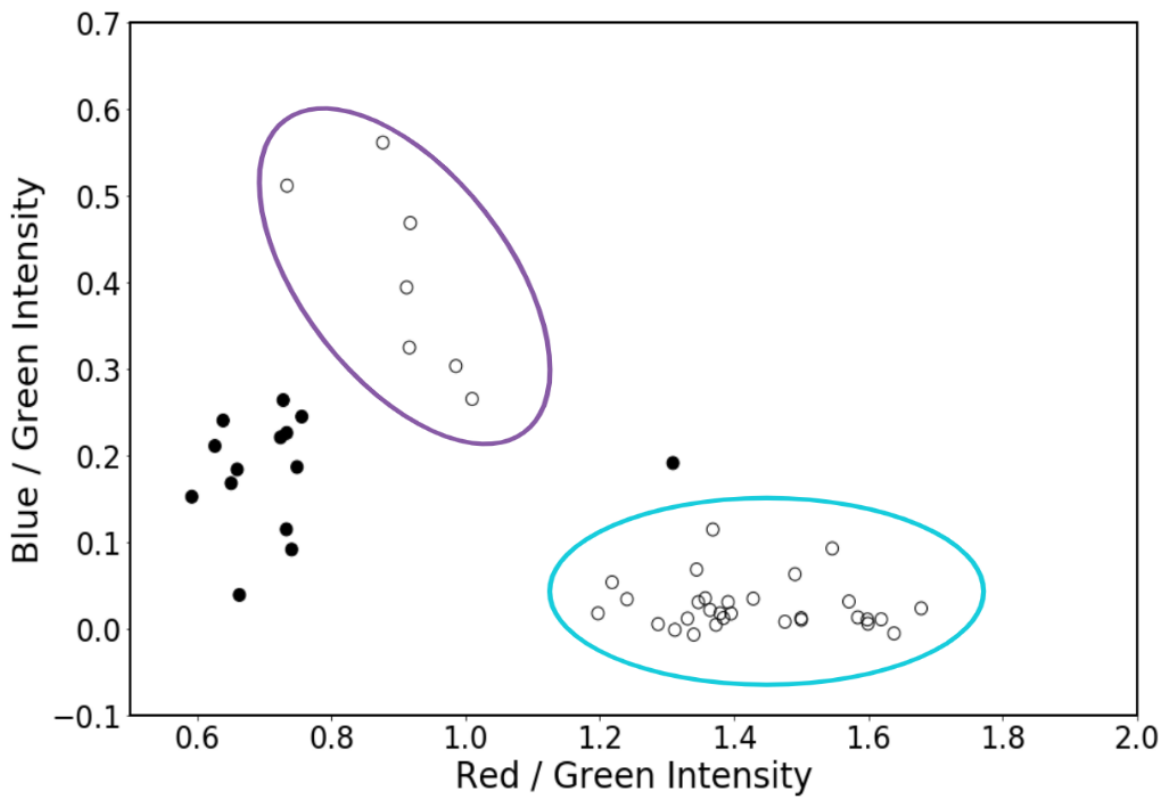

Figure 8: Blue/Green intensities versus Red/Green intensities for 60 sources selected from the towns of Monterey (open circles) and nearby Hartz Lake (filled circles). 
inspection of the locations of the sources on daylight maps confirms that within Monterey, all street lights cluster at the bottom right, while light sources associated with houses, farms, or commercial establishments, form the looser, but well-separated cluster centered around $(0.9,0.4)$.

While the Monterey/Hartz Lake results are suggestive that sources can be distinguished by lamp type, we do not have direct confirmation that the geographic distinction is associated with a lamp type distinction. We thus reanalyzed our images of the town of Culver, particularly focusing on a subset of light sources for which we have ground truthing data gathered on an expedition in April 2020. In this ground survey we located, identified and cataloged 56 distinct light sources found in the NITELite images in Culver and Monterey/Hartz Lake and mapped via visual identification using Google Earth imagery. This involved a daytime and follow up nighttime survey with a visual confirmation of the type of light sources. Figure 9 shows the Culver sources plotted by their red/green versus blue/green color ratios. Again, we see a clean separation into clusters. This time, however, our ground truth data reveals that the clusters are strongly correlated with the lamp type. A closer inspection of the data shows that the rare interlopers and outliers are sources where either our centroiding/source location routines failed or sources disappeared (switched off or became obscured?) during the overpass. Either situation leads to incorrect color ratios displacing the source in color/color space

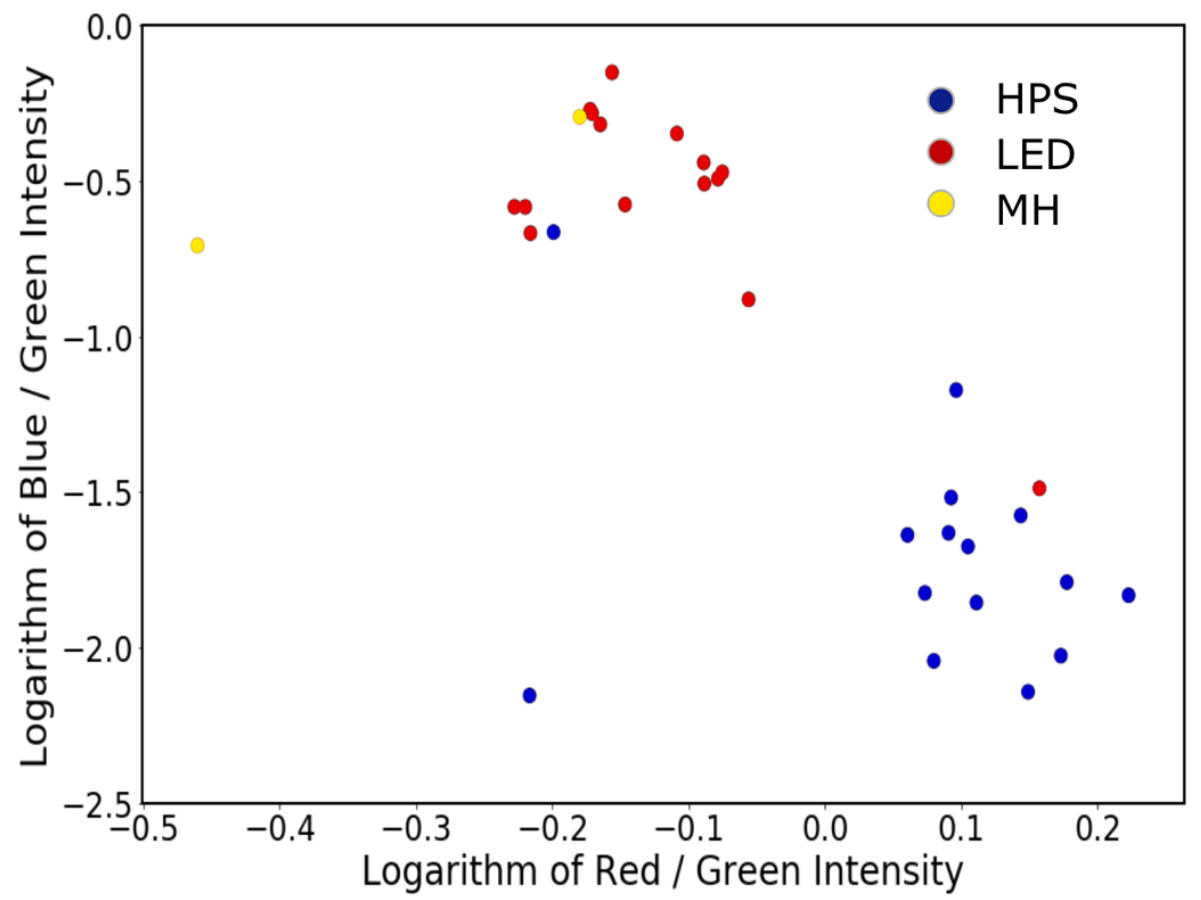

Figure 9: Red/Green intensity versus blue/green intensity for 32 sources in the town of Culver, IN. The sources are color coded according to lamp type - high-pressure sodium (HPS), LED and metal-halide (MH) - determined by a ground truthing survey conducted subsequent to the flight.

\section{Discussion (future prospects)}

The LHAB based NITELite system we have presented here opens the doors to a myriad of possible studies. Among them are:

1. A statistical study of the variation of radiated light as a function of angle from the zenith. While this quantity is of great importance in predicting the correlation between urban lights and the sky brightness, it has never been measured in detail.

2. A study of seasonal variations in emitted light or lighting retrofits. As vegetation (and snow/rain cover) changes, both direct obscuration and variations in reflection will affect the amount of light escaping to the atmosphere. Seasonal variations due to changes in human behavior may also be important. NITELite's low cost per mission makes following seasonal changes possible.

3. An improved understanding of short timescale lighting variations (minutes to hours) and mobile sources such as cars, trucks, etc. The ability of NITELite to dwell within range for minutes to hours allows repeated imaging of the same source within the space of a single night. 
Due to the COVID-19 pandemic, plans to perform flights over Chicago in 2020 had to be delayed. For similar reasons, a subsequent plan for a flight over Indianapolis, IN, in November 2020 was also cancelled. Future flight dates will be scheduled over Chicago and other areas once public safety conditions allow. In concert with these flights, we will be conducting coordinated ground observations via the deployment of our GONet (Ground Observation Network) system of all-sky cameras.

We are evaluating both hardware and software improvements in the NITELite system. Hardware-based improvements under consideration include a star tracker to provide much more precise pointing information, a fourth color to improve lamp type discrimination, and faster imaging to improve stacking (>1 image set/sec). Software enhancements include routines for stacking of images, a calibration pipeline, and a more fully automated analysis system. We are also in the early stages of exploring possible ways to automate the georegistration process, including utilizing neural net-based machine-learning to correlate between daylight and nighttime based images, and a citizen science system where we "outsource" this correlation to participants in the Zooniverse.org citizen science platform in a similar way that has been demonstrated by the Cities at Night project[34].

\section{References}

[1] Gibson, J., Olivia, S., \& Boe-Gibson, G. (2020). NIGHT LIGHTS IN ECONOMICS: SOURCES AND USES 1. Journal of Economic Surveys, 34(5), 955-980.

[2] Owens, A. C., Cochard, P., Durrant, J., Farnworth, B., Perkin, E. K., \& Seymoure, B. (2020). Light pollution is a driver of insect declines. Biological Conservation, 241, 108259.

[3] Nadybal, S. M., Collins, T. W., \& Grineski, S. E. (2020). Light pollution inequities in the continental United States: A distributive environmental justice analysis. Environmental research, 189, 109959.

[4] Rybnikova, N., \& Portnov, B. A. (2020). Testing the generality of economic activity models estimated by merging night-time satellite images with socioeconomic data. Advances in Space Research, 66(11), 2610-2620.

[5] Elvidge, C. D., Baugh, K. E., Kihn, E. A., Kroehl, H. W., \& Davis, E. R. (1997). Mapping city lights with nighttime data from the DMSP Operational Linescan System. Photogrammetric Engineering and Remote Sensing, 63(6), 727734.

[6] Xiao, Q., Gee, G., Jones, R. R., Jia, P., James, P., \& Hale, L. (2020). Cross-sectional association between outdoor artificial light at night and sleep duration in middle-to-older aged adults: the NIH-AARP Diet and Health Study. Environmental research, 180, 108823.

[7] Buxton, R. T., Seymoure, B. M., White, J., Angeloni, L. M., Crooks, K. R., Fristrup, K., ... \& Wittemyer, G. (2020). The relationship between anthropogenic light and noise in US national parks. Landscape Ecology, 35, 1371-1384.

[8] Kyba, C. C. M., Ruby, A., Kuechly, H. U., Kinzey, B., Miller, N., Sanders, J., ... \& Espey, B. (2020). Direct measurement of the contribution of street lighting to satellite observations of nighttime light emissions from urban areas. Lighting Research \& Technology, 1477153520958463.

[9] Greenhalgh, J., \& Meadows, K. (1999). Th use of patient-based measures of health in improving the process and outcomes of patien review. Journal of Evaluation in Clinical, 416.

[10] Bouroussis, C. A., \& Topalis, F. V. (2020). Assessment of outdoor lighting installations and their impact on light pollution using unmanned aircraft systems-The concept of the drone-gonio-photometer. Journal of Quantitative Spectroscopy and Radiative Transfer, 253, 107155.

[11] Fiorentin, P., Bettanini, C., \& Bogoni, D. (2019). Calibration of an autonomous instrument for monitoring light pollution from drones. Sensors, 19(23), 5091.

[12] Elvidge, C. D., Erwin, E. H., Baugh, K. E., Ziskin, D., Tuttle, B. T., Ghosh, T., \& Sutton, P. C. (2009, May). Overview of DMSP nightime lights and future possibilities. In 2009 Joint Urban Remote Sensing Event (pp. 1-5). IEEE.

[13] Hsu, F. C., Baugh, K. E., Ghosh, T., Zhizhin, M., \& Elvidge, C. D. (2015). DMSP-OLS radiance calibrated nighttime lights time series with intercalibration. Remote Sensing, 7(2), 1855-1876.

[14] Cinzano, P., Falchi, F., \& Elvidge, C. D. (2001). The first world atlas of the artificial night sky brightness. Monthly Notices of the Royal Astronomical Society, 328(3), 689-707.

[15] Elvidge, C. D., Baugh, K. E., Kihn, E. A., Kroehl, H. W., \& Davis, E. R. (1997). Mapping city lights with nighttime data from the DMSP Operational Linescan System. Photogrammetric Engineering and Remote Sensing, 63(6), 727 734.

[16] Elvidge, C. D., Baugh, K. E., Zhizhin, M., \& Hsu, F. C. (2013). Why VIIRS data are superior to DMSP for mapping nighttime lights. Proceedings of the Asia-Pacific Advanced Network, 35(0), 62.

[17] Lee, S., Chiang, K., Xiong, X., Sun, C., \& Anderson, S. (2014). The S-NPP VIIRS day-night band on-orbit calibration/characterization and current state of SDR products. Remote Sensing, 6(12), 12427-12446.

[18] Falchi, F., Cinzano, P., Duriscoe, D., Kyba, C. C., Elvidge, C. D., Baugh, K., ... \& Furgoni, R. (2016). The new world atlas of artificial night sky brightness. Science advances, 2(6), e1600377.

[19] Levin, N., Johansen, K., Hacker, J. M., \& Phinn, S. (2014). A new source for high spatial resolution night time images - The EROS-B commercial satellite. Remote Sensing of Environment, 149, 1-12.

[20] Li, X., Li, X., Li, D., He, X., \& Jendryke, M. (2019). A preliminary investigation of Luojia-1 night-time light imagery. Remote sensing letters, 10(6), 526-535.

[21] Pack, D., Hardy, B., \& Longcore, T. (2017). Studying the Earth at Night from CubeSats. 
[22] Zheng, Q., Weng, Q., Huang, L., Wang, K., Deng, J., Jiang, R., .. \& Gan, M. (2018). A new source of multi-spectral high spatial resolution night-time light imagery-JL1-3B. Remote sensing of environment, 215, 300-312.

[23] Castiglione, L., Conticello, S. S., Esposito, M., Oldenhuis, R., Moon, S. G., Nicolai, A., ... \& Dettmann, J. (2012, October). The NightPod-An orbital motion compensation mechanism for ISS based imaging. In Proceedings of the 63rd IAC (International Astronautical Congress), Naples, Italy.

[24] de Miguel, A. S., Kyba, C. C., Aubé, M., Zamorano, J., Cardiel, N., Tapia, C., ... \& Gaston, K. J. (2019). Colour remote sensing of the impact of artificial light at night (I): The potential of the International Space Station and other DSLR-based platforms. Remote sensing of environment, 224, 92-103.

[25] GYUK, G., GARCIA, J. G., TARR, C., \& WALCZAK, K. Light Pollution Mapping from a Stratospheric HighAltitude Balloon Platform.

[26] Kuechly, H. U., Kyba, C. C., Ruhtz, T., Lindemann, C., Wolter, C., Fischer, J., \& Hölker, F. (2012). Aerial survey and spatial analysis of sources of light pollution in Berlin, Germany. Remote Sensing of Environment, 126, 39-50.

[27] Hale, J. D., Davies, G., Fairbrass, A. J., Matthews, T. J., Rogers, C. D., \& Sadler, J. P. (2013). Mapping lightscapes: spatial patterning of artificial lighting in an urban landscape. PloS one, 8(5), e61460.

[28] Canty, T., Pickett, H. M., Salawitch, R. J., Jucks, K. W., Traub, W. A., \& Waters, J. W. (2006). Stratospheric and mesospheric HOx: Results from Aura MLS and FIRS-2. Geophysical research letters, 33(12).

[29] Crill, B. P., Ade, P. A., Artusa, D. R., Bhatia, R. S., Bock, J. J., Boscaleri, A., ... \& Turner, A. D. (2003). Boomerang: A balloon-borne millimeter-wave telescope and total power receiver for mapping anisotropy in the cosmic microwave background. The Astrophysical Journal Supplement Series, 148(2), 527.

[30] Pfotzer, G. (1972). History of the use of balloons in scientific experiments. Space Science Reviews, 13(2), $199-242$.

[31] Guzik, T. G., Ellison, S. B., Stewart, M., Wefel, J. P., Pierce, D., \& Garde, G. (2011, June). A Multiple Payload Carrier for High Altitude Ballooning. In Academic High Altitude Conference (Vol. 2011, No. 1). Iowa State University Digital Press.

[32] turbide. (2019, August 29). CRAQ researcher Martin Aubé and his students launch a balloon in the stratosphere to better understand light pollution. Center for Research in Astrophysics of Quebec (CRAQ). http://craqastro.ca/2019/08/29/craq-researcher-martin-aube-and-his-students-launch-a-balloon-in-the-stratosphere-to-betterunderstand-light-pollution/?lang=en

[33] Walczak, K., Gyuk, G., Kruger, A., Byers, E., \& Huerta, S. (2017). Nitesat: a high resolution, full-color, light pollution imaging satellite mission. International Journal of Sustainable Lighting, 19(1), 48-55.

[34] de Miguel, A. S., Castaño, J. G., Zamorano, J., Pascual, S., Ángeles, M., Cayuela, L., ... \& Kyba, C. C. (2014). Atlas of astronaut photos of Earth at night. Astronomy \& Geophysics, 55(4), 4-36. 